Témoigner Témoigner. Entre histoire et mémoire

Getuigen Revue pluridisciplinaire de la Fondation Auschwitz

$124 \mid 2017$

La musique dans les camps

\title{
Remember : Memento mori, victimes et bourreaux
}

Remember (Atom Egoyan)

\section{Gorik de Henau}

Traducteur : Emilie Syssau

\section{(2) OpenEdition}

\section{Journals}

Édition électronique

URL : https://journals.openedition.org/temoigner/5659

DOI : $10.4000 /$ temoigner.5659

ISSN : 2506-6390

Éditeur :

Éditions du Centre d'études et de documentation Mémoire d'Auschwitz, Éditions Kimé

Édition imprimée

Date de publication : 2 avril 2017

Pagination : $9-12$

ISBN : 978-2-930953-00-7

ISSN : 2037-4183

Référence électronique

Gorik de Henau, «Remember: Memento mori, victimes et bourreaux», Témoigner. Entre histoire et

mémoire [En ligne], 124 | 2017, mis en ligne le 30 novembre 2021, consulté le 01 décembre 2021. URL : http://journals.openedition.org/temoigner/5659 ; DOI : https://doi.org/10.4000/temoigner.5659

Tous droits réservés 


\section{REMEMBER : MEMENTO MORI, VICTIMES ET BOURREAUX}

CINÉMA Par son ingénieuse structure narrative, Remember, d'Atom Egoyan, déclenche une série de questions sur un passé chargé. Car, septante ans après, nous n'en avons toujours pas terminé avec l'héritage des camps nazis.

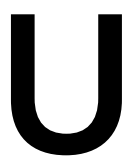
n homme âgé se réveille dans son lit et appelle sa femme. Elle n'est pas là. Au mur sont accrochés un chapeau de paille et une robe, qu'il caresse. L'homme ouvre la porte et demande où est sa femme. Il apparaît que nous sommes dans un endroit bien différent de celui que nous avions d'abord imaginé, car d'une chambre douillettement aménagée, on passe à l'environnement impersonnel d'une maison de retraite. Ce n'est pas la seule surprise que réserve Remember, un film qui prend le spectateur à contrepied.

Dans cette scène, nous faisons la connaissance de Zev. Il porte au cou une chaînette à laquelle pend une étoile de David, nous apprenons maintenant qu'il est juif. Peu après, on peut distinguer un tatouage sur son avant-bras gauche, un nombre à six chiffres. On comprend alors qu'il était détenu à Auschwitz-Birkenau, seul camp de concentration où les internés se voyaient infliger un tel code d'identification. Zev semble souffrir d'une forme sérieuse de démence. Mais ce n'est pas grave : son ami Max Rosenbaum a tout récapitulé dans une lettre qu'il lui a confiée. Ily décrit dans les moindres détails la tâche que Zev doit exécuter; chaque fois que celui-ci perd à nouveau la tête, il lui suffit de se référer à cette lettre.

Plusieurs nazis ont pris une nouvelle identité à la fin de la Seconde Guerre mondiale afin d'échapper aux poursuites et d'émigrer aux États-Unis sous un faux nom. L'homme responsable de la mort de leurs deux familles, le Blockführer Otto Wallisch, mène ainsi à présent sa vie sous le nom de Rudy Kurlander. Quatre hommes portent ce nom en Amérique du Nord. Zev doit aller les trouver un à un, déterminer si l'individu en face de lui est bien Wallisch et, si c'est le cas, l'assassiner. Tandis que Zev parcourt la liste, son fils entame des recherches. Nous sommes au quart du film, et avons fait connaissance avec les personnages les plus importants, nous connaissons le litige dramatique ainsi que la trame narrative : la liste. Nous voilà concernés.

Mais est-ce vraiment aussi simple qu'il semble à première vue? Revenons un peu en arrière et replaçons les choses dans un contexte cinématographique. L'association entre perte de mémoire et mission vengeresse était déjà la force motrice à l'œuvre dans Memento (Christopher Nolan, 2000), où un homme frappé d'amnésie part à la recherche des assassins de sa femme. Dans De zaak Alzheimer [La Mémoire du tueur] (Erik Van Looy, 2003), un tueur à gages frappé d'un début d'Alzheimer se retrouve impliqué dans un scandale pédophile. Généralement, le motif de la vengeance au cinéma a mauvaise réputation en raison de films réactionnaires avides de sensation comme Death Wish [Un justicier dans la ville] (Michael Winner, 1974). Mais •.•

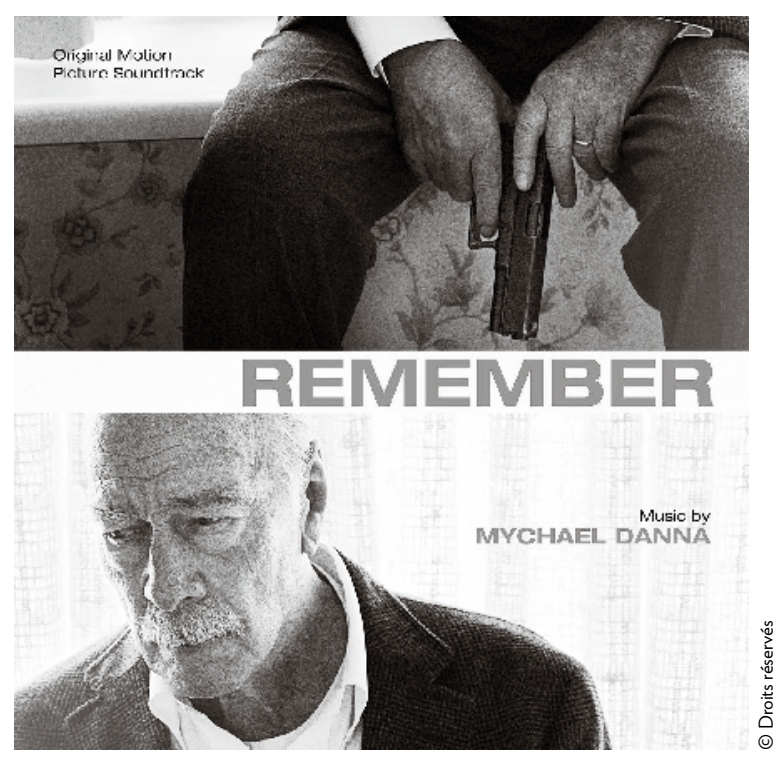


• • certains films «de vengeance» liés à la Shoah ont su se distinguer.

Dans Marathon Man (John Schlesinger, 1976), Dustin Hoffman s'attaque au chef (Laurence Olivier) d'u réseau de criminels nazis en fuite qui font du trafic de lamants provenant de victimes de guerre juives. This Must Be the Place (Paolo Sontentino, 2011) traitedune ancienter

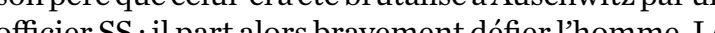
film uruguaren $M r$. Kaplan (Álvấ film uruguayen Mr. Kaplan (Alvaro Brechner, 2014) un âge avancé. Un octogénare juif y entreprens sur un âge avane. Un octogenaire juif y entreprend sur allemand qui n'est finalement pas le cristerieux qu'il avait en vue. Et, colones pas le chiminel nazi criminels de guerre japonis, citons The Railus des [Les Voies du destin] (Jonathan Teplitzky, 2013 ; nique dans le numéro 122 de Témoigner. Entre histoire

et mémoire), où un ancien prisonnier de guerre britanencen à son ancien bou et renonce à ses projets de vengeance.

La réalisation est cette fois du cinéaste canadien Atom Egoyan, qui se penche pour la première fois sur le thème de la mémoire juive. Surtout connu pour ses films traitant des problèmes de communication dan une société déboussolée (Family Viewing, Exotica, The Sweet Hereafter [De beaux lendemains]), il a ensuite tourné deux films dans lesquels il aborde la mémoir du génocide arménien en 1915, du point de vue des génératide arménien en 1915, du point de vue des genérations nées dur (1993) dia diaspora. Le film experipastal Calendar (19g3) entrenele une commande passée à un photographe (prendre des photos deglise rat (2002) selon vertains le premier fil-i. Dans Ararat (2002), selon certains le premier film anglophone suŕ le génocide arménien, Egoyan montre en quoi la mémorepentparfoisêtrepefideen forctionc

des personnes concernées.
Remember renferme aussi une problématisation similaire de la vérité unique. En littérature, les auteurs tempirent louventla revendication d'athenticién créant un narrateur suspectqui donne au lecteur une crion ber, la posture narrative est tou farts. Dans Remem-

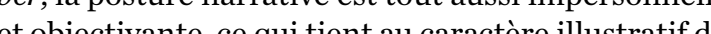

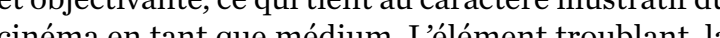
cettre, s'entrelace ici à l'intrigue. Par commladité,

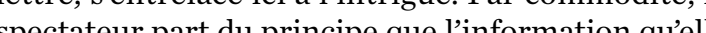
spectateur part du pring que linformation quelle content certain. Tout bin naré de façonsór

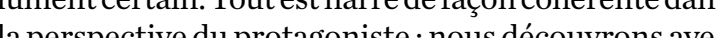
lui le contive duprotagoniste; nous découvrons avec tion extériere qu nous permettrat d'en verifer tín extérier

Par moments, le feu vacillant de la mémoire de Ainsi, il réagit en entendant la pluie tambouriner sur
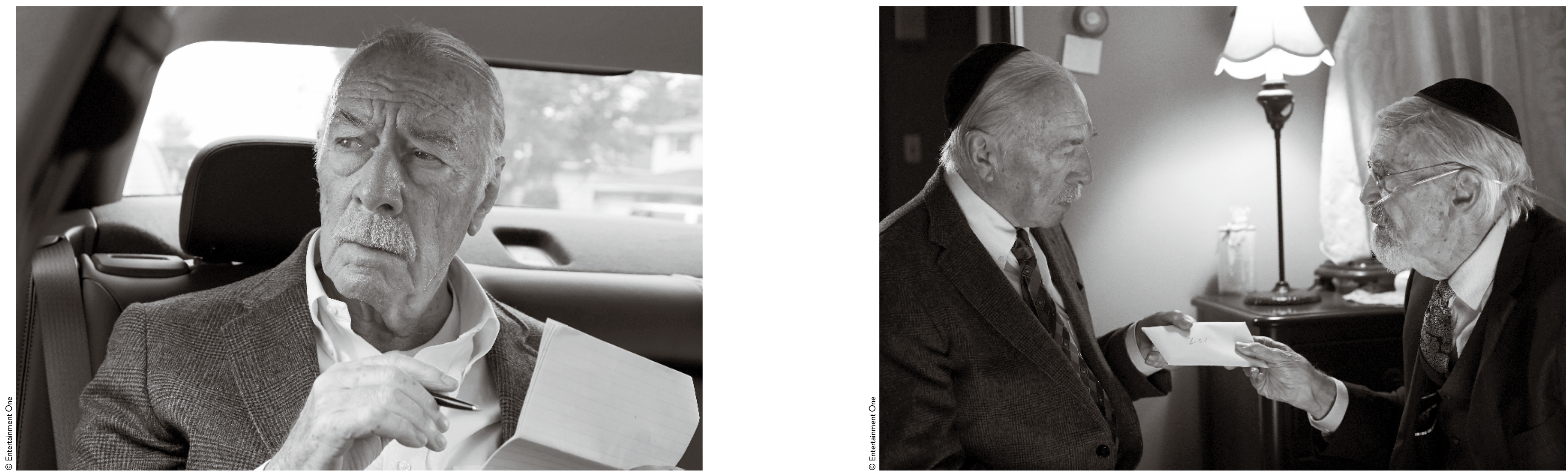


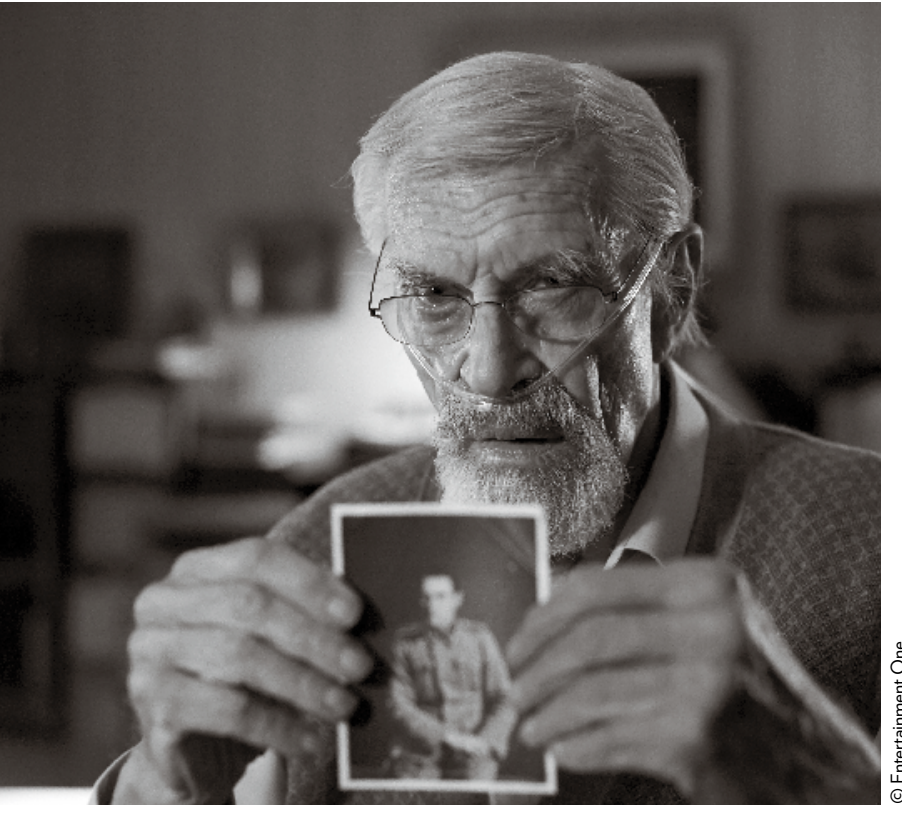

•• ses pérégrinations, Zev tombe sur un piano et se met spontanément à jouer la musique de sa jeunesse - souvenirs d'un passé dénué de problème, qui n'était pas contaminé par son état médical. Il dit à un auditeur admiratif : «My piano teacher told me the three most important composers are Mendelssohn, Meyerbeer en Moszkowski » [Mon professeur de piano m'a dit que les trois compositeurs les plus importants étaient Mendelssohn, Meyerbeer et Moszkowski] - tous trois, notons-le, sont d'origine juive.

Sur le plan visuel, on note l'utilisation ambivalente du motif de l'étoile, à travers le pendentif en étoile de David au cou de Zev, dans l'insigne d'un policier antisémite et comme logo d'une compagnie de bus. Avec ces subtiles allusions au judaïsme et à la Shoah, Egoyan joue en permanence avec le spectateur.

Remember est le premier scénario filmé du quasi inconnu Benjamin August. D’origine juive, il n’aurait, selon Wikipedia, perdu aucun parent direct lors de la Shoah. L’intrigue serait par ailleurs imprégnée de son expérience de professeur d'anglais au Vietnam, où il a été amené à réfléchir à l'héritage (souvent violent) laissé par l'armée américaine dans ce pays. Scénariste et réalisateur présentent l'intrigue dans les règles de l'art et entretiennent l'obscure tension jusqu'à la fin. Mais on s'interroge sur leurs intentions.

La dernière génération des rescapés des camps de concentration nazis s'éteint lentement, la poignée de survivants est maintenant très âgée. Au moment où la source constituée par les informations de première main menace de se tarir, la société arrive à un point charnière : que faire de cet héritage ? Dans quelle mesure avons-nous tiré les leçons du passé ? Va-t-on tourner la page et faire table rase, avec le risque de reproduire les mêmes erreurs? Remember menace de subordonner toute cette problématique à une intrigue captivante, qui, conformément aux consignes des manuels de scénario américains, contient un tournant inattendu remettant en question tout ce qui précède.

Il existe un nom pour ce type de récits faciles à prétexte historique : exploitation. Remember n'a en effet rien à dire sur les événements survenus dans les camps ni sur l'interaction entre Blockführer et détenus, sur laquelle le film assied cependant sa légitimité. Et, si on y réfléchit à froid, l'hypothèse même du film, que nous ne dévoilerons pas ici, est invraisemblable (ce qui est certes le cas de nombreux thrillers et autres films à intrigue). Remember semble donc davantage contribuer au débat sur l'absurdité ou non du thème de la mémoire, le film ayant le mérite de présenter ce phénomène au grand public.

Gorik de Henau

Mémoire d'Auschwitz ASBL Traduit du néerlandais par Emilie Syssau

\section{$\longrightarrow$ Plus d'infos \\ $\rightarrow$ Remember d'Atom Egoyan (2015)}

Avec: Christopher Plummer (Zev Guttman), Martin Landau (Max Rosenbaum), Jürgen Prochnow (Rudy Kurlander n ${ }^{\circ} 4$ ) e.a. -94 minutes.

\section{Wikipedia, entrée Benjamin August,}

https://en.wikipedia.org/wiki/Benjamin_August, consulté le 11 septembre 2016.

$\Leftrightarrow$ Jordan Adler, «Movie about Nazi war criminals opens this week », The Canadian Jewish News, 22 octobre 2015, http:// www.cjnews.com/culture/entertainment/movie-about-nazi-warcriminals-opens-this-week, consulté le 11 septembre 2016. 